\title{
Kontinenz- und Stomaversorgung
}

\author{
Qualität sicherstellen
}

Ziel einer Rehabilitation ist die Wiedererlangung des körperlichen, seelischen und sozialen Wohlbefindens. Wenn eine bleibende Beeinträchtigung des normalen Lebens vorliegt, wie dies bei einer Stomaanlage der Fall ist, ist die Notwendigkeit einer umfassenden rehabilitativen Betreuung besonders bedeutsam. Entsprechende Qualitätskriterien sollen sicherstellen, dass der Betroffene sein Leben mit möglichst geringen Einschränkungen und hoher Lebensqualität führen kann. Die Österreichischen Kontinenz- und Stomaberater haben gemeinsam mit dem Verband Österreichischer Stomatherapeuten und der Medizinischen Kontinenzgesellschaft mit dem im vergangenen Jahr verabschiedeten Positionspapier die Tätigkeiten und Aufgaben der Kontinenz- und Stomaberatung festgehalten und möchten damit einen bundesweit einheitlichen hohen Qualitätsstandard für Kontinenz- und Stomaberater in Ausbildung und Praxis festlegen. Zum Wohle des Patienten und als Orientierungshilfe für die ausgebildeten Kontinenz- und Stomaberater.

\section{Komplexes Bild}

Der Betroffene ist nach der Stomaanlage in einer völlig neuen Lebenssituation, die nicht nur durch die Stomaanlage selbst und die Notwendigkeit ihrer Versorgung verursacht ist, sondern auch durch belastende perioperative Begleitumstände. So geben mehr als 80 Prozent der Patienten nach der Operation akute Beschwerden an, die sich in Form von verzögerter Wundheilung, Schmerzen, Harninkontinenz oder auch Komplikationen im Bereich der Stomanlage manifestieren. Die Belastung der Operation erschwert jedoch in vielen Fällen die Umsetzung rehabilitativer Maßnahmen in der unmittelbar postoperativen Phase, da die Patienten zumeist nur eingeschränkt aufnahmefähig sind. Nicht zuletzt aufgrund der psychischen Belastung. In dieser Zeit soll der Stomapatient lernen das Stoma zu versorgen, mit dem Versorgungsmaterial umzugehen, sich Wissen über Komplikationen und vorbeugende Maßnahmen aneignen und, wenn notwendig, eine Stuhlregulierung durchführen. In den meisten Fällen ist der Patient damit überfordert. Allerdings haben Erfahrungen und auch diesbezügliche Untersuchungen gezeigt, dass eine entsprechende fachliche Schulung durch speziell ausgebildete Stomatherapeuten bereits vor der Operation viele dieser Hindernisse überwenden kann (siehe PROCARE 07-08/2008).

Eine umfassende speziell auch die seelischen Bedürfnisse des Patienten einschließende Beratung im Anschluss an die Stomaanlage sollte die psychisch-soziale Integration, die Akzeptanz verbleibender Restbehinderungen, die Verminderung der Lebensqualität und berufliche bzw. wirtschaftliche Belastungen erfassen und Lösungsangebote geben.

\section{Bedarfsgerechte Struktur}

Zur optimalen Rehabilitation von Stomaträgern bedarf es einer bedarfsgerechten Struktur, die bestimmte Voraussetzungen erfüllen sollte. So sollten die Ärzte entsprechende Zusatzkenntnisse aufweisen und ebenso wie die Krankenpflegepersonen ausreichende Erfahrung in der Stomaversorgung haben, spezielle Mitarbeiter sollten über eine zusätzliche Praxis in der Kostund Ernährungsberatung und problemorientierte Erfahrung über psychische, soziale und berufliche Besonderheiten verfügen. Es sollte möglich sein, alle auf dem Markt befindlichen Versorgungsmöglichkeiten bereitzustellen, sowie ausreichende Räumlichkeiten und Personal anzubieten.

Für die Qualität des Rehabilitationsprozesses gilt die Durchführung verschiedener Maßnahmen als Maßstab. Dazu zählen das zügige Erlernen einer sicheren Stomaversorgung, das Erlernen der Irrigation, falls möglich, der Erwerb von Kenntnissen in der Beurteilung und eventuellen Behandlung von Stomakomplikationen, Sicherheitstraining in Bereichen wie Schwimmen und Gymnastik, Information über eventuell notwendige Stuhlregulierung, und über die Nachsorge bei Tumorkrankheiten falls notwendig, psychische
Hilfestellung und Beratung bei sexuellen Problemen ebenso wie soziale Beratung, Organisation der ambulanten Versorgung und umfassende Kommunikation. Ziel der Stomapflege ist die Selbstständigekeit und Unabhängigkeit des Stomaträgers. Dazu zählt auch wesentlich die umfassende neutrale Produktauswahl von Stomaartikeln und weiteren Hilfsmitteln. Nur durch die Produktvielfalt ist es für den Stomaträger möglich eine sichere, individuelle Stomaversorgung zu erhalten.

Entsprechend all dieser notwendigen Informationsebenen ist die Fortbildung der Stoma- und Kontinenzberater inhaltlich gestaltet. Alle standardisierten Vorgangsweisen müssen naturgemäß auf die Bedürfnisse des Patienten abgestimmt sein, müssen also genügend Flexibilität für dessen Individualität haben.

\section{Kontinenzzentren}

„Die Stoma- und Kontinenzberatung ist ein gutes Beispiel für die breite interdisziplinäre Zusammenarbeit", stellte Univ.-Prof. Dr. Helmut Madersbacher, Univ-Klinik Innsbruck, anlässlich der Jahrestagung der Medizinischen Kontinenzberatung im vergangenen Oktober in Linz fest. So ist auch die Initiative der MKÖ zur Zertifizierung von Kontinenz- und Beckenbodenzentren zu verstehen. Die Anforderungen an derartige Kompetenzzentren sind die Zusammenarbeit der drei Kerndisziplinen Urologie, Gynäkologie und Chirurgie, wobei jedoch mindesten fünf voneinander unabhängige Fachdisziplinen vereint sein sollen, die sich schwerpunktmäßig mit der Thematik befassen - unter Einbindung des Hausarztes ambulanter Pflegedienste, Reha-Einrichtungen und Selbsthilfegruppen.

Eine weitere Initiative des MKÖ ist die Verleihung eines Gütesiegels für Produkte der Kontinenzversorgung, die dem Anwender Sicherheit geben sollen, ein hochwertiges Produkt anzuwenden. Für die Krankenkassen könnte dieses Gütesiegel eine Hilfestellung in der Entscheidung für Kostenerstattungen darstellen. 